Review Article:

\title{
Reducing Unnecessary CT Scanning in Head Trauma of Pediatric Patients: A Narrative Review
}

\author{
Alireza Baratloo ${ }^{1}$ (D), Mohammad-Taghi Talebian² (D), Sahar Mirbaha ${ }^{3}$ (D), Shahram Bagheri-Hariri² ${ }^{*}$ (D)
}

1. Department of Emergency Medicine, Sina Hospital, Tehran University of Medical Sciences, Tehran, Iran.

2. Department of Emergency Medicine, Imam Khomeini Hospital Complex, Tehran University of Medical Sciences, Tehran, Iran.

3. Department of Emergency Medicine, Shohaday-e-Tajrish Hospital, Shahid Beheshti University of Medical Sciences, Tehran, Iran.

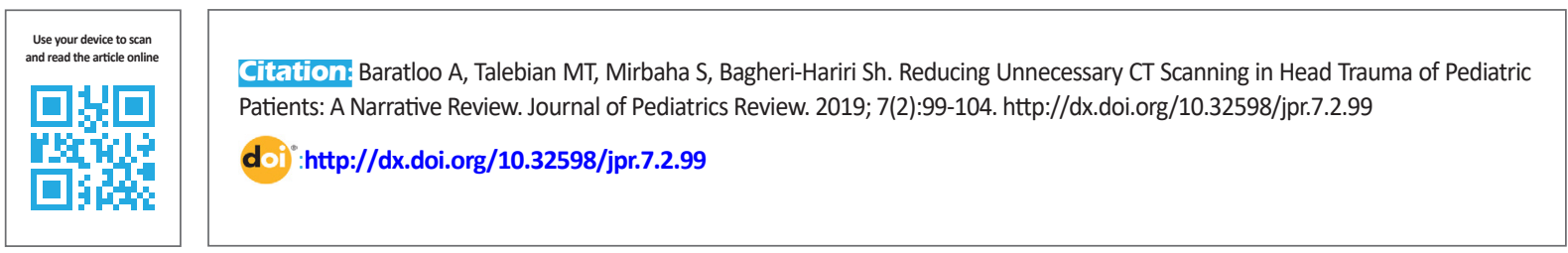

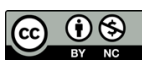

Funding: See Page 102

Article info:

Received: 28 January 2018

First Revision: 20 February 2018

Accepted: 29 May 2018

Published: 01 April 2019
Keywords:

Decision support

techniques, Closed head injuries, Pediatrics, X-ray computed tomography, Unnecessary procedures

\begin{abstract}
A B STR A C T
Context: The current review article considered the latest published papers on reducing unnecessary head Computed Tomography (CT) scans in pediatric patients, to pave the way for further surveys.

Evidence Acquisition: The papers were selected through Google Scholar and PubMed. We searched "reducing unnecessary head CT scan in pediatric patients" with related keywords compatible with MeSH system in accordance with the search strategy. Original articles and systematic reviews published after 2010 were included. Other types of manuscripts such as a letter to editor, editorial reviews, case series, etc. were excluded. The title and abstract of the eligible articles were assessed in terms of relevance to our topic. The full text of final selected papers were studied by the investigators. Furthermore, a critical appraisal was performed in an expert panel to summarize it and make it applicable.

Results: The extracted manuscripts have addressed this issue by various strategies. The findings could be categorized as follows: observation period, focused history taking, protocol and guideline development, implementation of validated clinical prediction rules, and blood-based decision making by assessing neuro-biomarker levels.

Conclusions: Most of the reviewed articles were focused on clinical findings to discover the minimum or low risk category of the pediatric patients with blunt head trauma. Other articles attempted on facilitating the use of available clinical prediction rules in this regard. Also, the measurement of serum biomarker levels has been considered; however, their widely application in practice is not well supported by evidence yet.
\end{abstract}

\section{Context}

rauma is definitely among the most frequent reasons for admission to Emergency Department (ED). Pediatric patients consist a signifi- cant part of ED trauma patients, with special and different needs from adults. Physical examination is rather difficult in this age group, and decision making depends on the use of radiology. Head Computed Tomography

* Corresponding Author:

Shahram Bagheri-Hariri, MD.

Address: Department of Emergency Medicine, Imam Khomeini Hospital Complex, Tehran University of Medical Sciences, Tehran, Iran. Tel: +98 (912) 1324877

E-mail: hariri.shahram@gmail.com; hariri@sina.tums.ac.ir 
(CT) scan is frequently used in this regard, but various physicians assessed its interpretation accuracy $(1,2)$.

CT scan is regularly used as a high sensitive test to rule out the intracranial and skull injuries following trauma in children. Its rate of use varies in different countries and even different health centers within a country (3-5). Considering the high rate of normal imaging, it is necessary to conduct studies to reduce unnecessary head CT scanning and excessive radiation exposure, in this population. This topic has also drawn much attention, because of many known or even unknown short- or long-term side effects of imaging $(6,7)$. The current study aimed to review the latest published papers on this subject to develop a basic structure for improving surveys.

\section{Evidence Acquisition}

The relevant papers were selected searching Google Scholar and PubMed. We searched papers on "reducing unnecessary head CT scan in traumatic pediatric patients" with related keywords compatible with MeSH system in accordance with the search strategy. The selected keywords were tomography, X-ray computed, pediatrics, unnecessary procedures, head injuries, and closed.

Papers with the main aim of reducing the number of head CT scan in trauma pediatric patients were considered eligible. Original articles and systematic reviews, published after 2010 were included. Other types of manuscripts such as letter to the editor, editorial reviews, case series, etc. were excluded. Papers in languages other than English were also excluded. By reviewing the articles, duplicates were omitted using Endnote software.

The title and abstract of the eligible articles were assessed in respect of relevance to our topic. The full-text of selected papers were studied by the investigators. Furthermore, a critical appraisal was performed in an expert panel to summarize it and make it applicable. Finally, the extracted subjects were categorized in the following section and the manuscript was prepared. Then, the complete manuscript was reviewed, revised and approved by all of the authors.

\section{Results}

Researchers are trying to implement different strategies to reduce the number of unnecessary head CT scans in pediatric patients. Their findings are categorized and discussed in the following subheadings. There were specific answers to the main question of this review article. The extracted manuscripts have addressed this issue with various strategies that are summarized below.

\subsection{Observations and reassessment before decision making}

Nigrovic et al. conducted a prospective multicenter observational study to evaluate an observation period prior to make decision on the necessity of head CT scan in pediatric patients with minor blunt head trauma. They reported that their mentioned strategy was associated with reduced number of head CT scanning (8).

Most Emergency Department (ED) physicians prefer to perform head CT scans in response to the worried parents and decide on the patient's condition, at the soonest possible. Overcrowded EDs, and lack of proper and specific place for pediatric observation can also influence such preference. However, considering a period for observation and reassessment could be effective in reducing unnecessary head CT scans in pediatric patients (8).

\subsection{Medical history, suggestive but not conclusive}

Palchak et al. examined whether an isolated history of Loss of Consciousness (LOC) and or amnesia is predictive of TBI (Traumatic Brain Injury) in pediatrics affected by blunt head trauma. They concluded that such history, without other clinical findings, was not accompanied with either pathologic findings in head CT scan or with any TBI (9). Another study by Palchak et al. examined the history of posttraumatic vomiting in a child as a considerable risk factor for TBI and concluded that such history could not prevent the risk for TBI (10).

\subsection{Guideline or protocol development}

Astrand et al. published the Scandinavian guideline for primary management of children affected by head trauma. The guideline categorized the head trauma pediatric patients into minimal, mild and moderate. They also divided the mild group into 3 subcategories including high-risk, medium-risk and low-risk groups. Based on this guideline, we can refrain from performing head CT scan in children with minimal risk, mild low-risk and mild moderate-risk head trauma (11).

Protocols were commonly used as a local solution in response to the needs of specific centers, especially the educational ones, in which the physicians in charge including residents' shifts are changed regularly. The protocols were modified by the physicians of the related center, despite being evidence-based. Guidelines are usually prepared by national or international scientific 
forums and are constructed using high levels of evidence from an accurate literature review.

\subsection{Implementation of clinical prediction rules}

Osmond et al. (2010) developed a clinical decision rule for the management of pediatric patients with blunt head trauma. Failure to reach score 15 on the Glasgow Coma Scale (GCS) within 2 hours, suspected open skull fracture, worsening headache and irritability were considered as high-risk factors with a $100 \%$ sensitivity for the necessity of further investigations. Large, boggy hematoma of the scalp, signs of basal skull fracture, dangerous mechanism of injury were considered as medium risk factors with $98.1 \%$ sensitivity for the prediction of TBI by head CT scan. This clinical prediction rule is called "CATCH" (Canadian Assessment of Tomography for Childhood Head Injury) (12). Jennings et al. evaluated the impact of a validated clinical prediction rule on decreasing the use of head CT scan in children. They reported that the rate of performing head CT scan decreased significantly from $29.2 \%$ to $17.4 \%$ when using this prediction rule (13).

Derivation of clinical prediction rules has attracted a lot of attention in recent years. In such surveys, patients at low risk or very low risk were identified following a prospective high power cohort study, to be excluded from further unnecessary diagnostic services. The National Institute for Health and Care Excellence (NICE) in 2004, The Royal College of Surgeons of England (RCS) in 2005, Children's Head injury Algorithm for the prediction of Important Clinical Events (CHALICE) in 2006, and Canadian Assessment of Tomography for Childhood Head injury (CATCH) in 2010, developed their guidelines for an optimal management of pediatric population referring to ED due to head trauma $(12,14-16)$. However, the PECAREN (Pediatric Emergency Care Applied Research Network) study was a valuable study on reducing unnecessary pediatric head CT scan, which developed and validated an age-specific clinical prediction rule for the children younger and older than 2 years of age, separately (17).

Normal mental status, no presence of scalp hematoma (except frontal), no loss of consciousness or loss of consciousness for less than 5 seconds, non-severe injury mechanisms, no palpable skull fracture, and normal behavior according to the parents obviate the need for performing head CT in children younger than 2 years old, with Negative Predictive Value (NPV) of $100 \%$. Also, normal mental status, no loss of consciousness, no vomiting, non-severe injury mechanisms, no signs of basilar skull fracture, and no severe headache obviate the need for performing head CT in children older than 2 years with NPV of $99.95 \%$ (17). There is no consensus on the most valuable clinical prediction rules, and research in this area is still on-going (18).

Ghizoni et al. performed a critical appraisal on the literature and their final conclusion was in favor of accepting the PECARN guidelines. This guideline suggests head CT scans for children with GCS of 14, altered level of consciousness and palpable skull fracture, or when warranted by the physician experience, the presence of multiple findings or worsening symptoms (19).

\subsection{Gadgets for ease of use}

Atabaki et al. reported that despite introducing the validated evidence-based clinical prediction rules, the rate of unnecessary head CT scans had not decreased. Thus, they designed an electronic health record (EHR) for easier application (20). Using computerized clinical decision support systems, web-based calculator, or mobile applications facilitate the use of clinical prediction rules $(21,22)$.

\subsection{Blood-based decision making: Neuro-biomarkers}

Langness et al. investigated the role of measurement of plasma D-dimer level to decrease unnecessary head CT scans in children affected by blunt head trauma. They reported that plasma D-dimer level less than $750 \mathrm{pg} / \mu \mathrm{L}$ could significantly preclude the number of unnecessary head CT scans (23).

Papa et al. investigated the possible role of serum levels of Ubiquitin C-terminal Hydrolase (UCH-L1) in predicting pathologic findings on head CT scan in children with mild and moderate TBI with GCS of 15 . They reported a sensitivity and NPV of $100 \%$, but specificity of $47 \%$ for a UCH-L1 cut-off level of $0.18 \mathrm{ng} / \mathrm{mL}$ (24).

Manzano et al. evaluated the possible role of serum S100B level in predicting the TBI in pediatrics population affected by mild blunt head trauma. They concluded that serum S100B level measurement in the first 6 hours of trauma with a cut-off point of $0.14 \mu \mathrm{g} / \mathrm{L}$ would have a sensitivity of $95 \%$. Therefore, it could be used to reduce unnecessary head CT scans (25).

Numerous researchers recommend the measurement of some biomarkers in blood sample, as a reliable method to predict possible TBI (26). S100B protein has been frequently assessed in different studies to find its best 
cut-off point. However, there is no consensus on the best time period for sampling S100B protein. Considering the available literature, S100B has a high sensitivity but low specificity for predicting $\mathrm{TBI}$ in children $(25,27)$. UCH-L1, D-dimer, Myelin Basic Protein (MBP), Neuron-Specific Enolase (NSE), Glial Fibrillary Acid Protein (GFAP), interleukin-6 and interleukin-8 have also been investigated to some extent in this regard. However, further evaluations are required before practicing their application $(23,24,28)$. Such biomarkers have been investigated solely, and it may be logical to investigate them in combination in this regard.

\section{Conclusions}

We mostly reviewed studies focused on clinical findings to discover the very low risk or low risk category of the pediatric patients with blunt head trauma. Other articles attempted on facilitating the use of available clinical prediction rules in this regards. Furthermore, the measurement of serum biomarker levels has been considered; however, their widely application in practice is not well supported by evidence yet.

\section{Ethical Considerations}

\section{Compliance with ethical guidelines}

There is no ethical principle to be considered doing this research.

\section{Funding}

This research did not receive any specific grant from funding agencies in the public, commercial, or not-forprofit sectors.

\section{Authors contributions}

All authors passed four criteria for authorship contribution based on recommendations of the International Committee of Medical Journal Editors (ICMJE).

\section{Conflict of interest}

The authors declare no conflict of interest.

\section{References}

1. Talebian MT, Kavandi E, Farahmand S, Shahlafar N, Arbab $M$, Seyedhosseini Davarani $S$, et al. Comparing the brain $\mathrm{CT}$ scan interpretation of emergency medicine team with radiologists' report and its impact on patients' outcome. Emergency Radiology. 2015; 22(3):261-8. [DOI:10.1007/ s10140-014-1279-0] [PMID]

2. Dolatabadi AA, Baratloo A, Rouhipour A, Abdalvand A, Hatamabadi $H$, Forouzanfar $M$, et al. Interpretation of computed tomography of the head: Emergency physicians versus radiologists. Trauma Monthly. 2013; 18(2):86-9. [DOI:10.5812/traumamon.12023] [PMID] [PMCID]

3. Stanley RM, Hoyle JD, Jr., Dayan PS, Atabaki S, Lee L, Lillis $\mathrm{K}$, et al. Emergency department practice variation in computed tomography use for children with minor blunt head trauma. The Journal of Pediatrics. 2014; 165(6):1201-6. [DOI:10.1016/j.jpeds.2014.08.008] [PMID]

4. Blackwell CD, Gorelick M, Holmes JF, Bandyopadhyay S, Kuppermann N. Pediatric head trauma: Changes in use of computed tomography in emergency departments in the United States over time. Annals of Emergency Medicine. 2007; 49(3):320-4. [DOI:10.1016/j.annemergmed.2006.09.025] [PMID]

5. Yoshida K, Krille L, Dreger S, Hoenig L, Merzenich H, Yasu $\mathrm{K}$, et al. Pediatric computed tomography practice in Japanese university hospitals from 2008-2010: Did it differ from German practice? Journal of Radiation Research. 2017; 58(1):135-41. [DOI:10.1093/jrr/rrw074] [PMID] [PMCID]

6. Pearce MS, Salotti JA, Little MP, McHugh K, Lee C, Kim KP, et al. Radiation exposure from CT scans in childhood and subsequent risk of leukaemia and brain tumours: A retrospective cohort study. The Lancet. 2012; 380(9840):499-505. [DOI:10.1016/S0140-6736(12)60815-0]

7. Brenner D, Elliston C, Hall E, Berdon W. Estimated risks of radiation-induced fatal cancer from pediatric CT. American Journal of Roentgenology. 2001; 176(2):289-96. [DOI:10.2214/ajr.176.2.1760289] [PMID]

8. Nigrovic L, Schunk J, Foerster A, Cooper A, Miskin M, Atabaki $S$, et al. The effect of observation on cranial computed tomography utilization for children after blunt head trauma. Pediatrics. 2011; 127(6):1067-73. [DOI:10.1542/ peds.2010-3373] [PMID]

9. Palchak MJ, Holmes JF, Vance CW, Gelber RE, Schauer BA, Harrison MJ, et al. Does an isolated history of loss of consciousness or Amnesia predict brain injuries in children after blunt head trauma? Pediatrics. 2004; 113(6):e507-13. [DOI:10.1542/peds.113.6.e507] [PMID]

10. Palchak MJ, Holmes JF, Vance CW, Gelber RE, Schauer BA, Harrison MJ, et al. A decision rule for identifying children at low risk for brain injuries after blunt head trauma. Annals of Emergency Medicine. 2003; 42(4):492-506. [DOI:10.1067/ S0196-0644(03)00425-6]

11. Astrand R, Rosenlund C, Undén J. Scandinavian guidelines for initial management of minor and moderate head trauma in children. BMC Medicine. 2016; 14:33. [DOI:10.1186/ s12916-016-0574-x] [PMID] [PMCID]

12. Osmond MH, Klassen TP, Wells GA, Correll R, Jarvis A, Joubert $\mathrm{G}$, et al. CATCH: A clinical decision rule for the use of 
computed tomography in children with minor head injury. Canadian Medical Association Journal. 2010; 182(4):341-8. [DOI:10.1503/cmaj.091421] [PMID] [PMCID]

13. Jennings R, Burtner J, Pellicer J, Nair D, Bradford $M$, Shaffer $M$, et al. Reducing head CT Use for children with head injuries in a community emergency department. Pediatrics. 2017; 139(4):e20161349. [DOI:10.1542/peds.2016-1349] [PMID]

14. Dunning J, Daly JP, Malhotra R, Stratford Smith P, Lomas J, Lecky F, et al. The implications of NICE guidelines on the management of children presenting with head injury. Archives of Disease in Childhood. 2004; 89(8):763-7. [DOI:10.1136/adc.2003.042523] [PMID] [PMCID]

15. Dunning J, Daly J, Lomas J, Lecky F, Batchelor J, Mackway Jones K. Derivation of the children's head injury algorithm for the prediction of important clinical events decision rule for head injury in children. Archives of Disease in Childhood. 2006; 91(11):885-91. [DOI:10.1136/adc.2005.083980] [PMID] [PMCID]

16. Dunning J, Daly J, Lomas J, Lecky F, Batchelor J, Mackway Jones K. Derivation of the children's head injury algorithm for the prediction of important clinical events decision rule for head injury in children. Archives of Disease in Childhood. 2006; 91(11):885-91. [DOI:10.1136/adc.2005.083980] [PMID] [PMCID]

17. Kuppermann N, Holmes JF, Dayan PS, Hoyle JD, Atabaki SM, Holubkov R, et al. Identification of children at very low risk of clinically-important brain injuries after head trauma: A prospective cohort study. The Lancet. 2009; 374(9696):116070. [DOI:10.1016/S0140-6736(09)61558-0]

18. Babl FE, Lyttle MD, Bressan S, Borland M, Phillips N, Kochar A, et al. A prospective observational study to assess the diagnostic accuracy of clinical decision rules for children presenting to emergency departments after head injuries (protocol): The Australasian Paediatric Head Injury Rules Study (APHIRST). BMC Pediatrics. 2014; 14:148. [DOI:10.1186/1471-2431-14-148] [PMID] [PMCID]

19. Ghizoni E, Fraga AMA, Baracat ECE, Joaquim AF, Fraga GP, Rizoli $S$, et al. [Indications for head computed tomography in children with mild traumatic brain injury (Portuguese)]. Ree vista do Colégio Brasileiro de Cirurgiões. 2013; 40(6):515-9. [DOI:10.1590/S0100-69912013000600016] [PMID]

20. Atabaki SM, Jacobs BR, Brown KM, Shahzeidi S, Heard Garris NJ, Chamberlain MB, et al. Quality improvement in pediatric head trauma with PECARN rules implementation as computerized decision support. Pediatric Quality \& Safety. 2017; 2(3):e019.

21. Dziadzko MA, Gajic O, Pickering BW, Herasevich V. Clinical calculators in hospital medicine: Availability, classification, and needs. Computer Methods and Programs in Biomedicine. 2016; 133:1-6. [DOI:10.1016/j.cmpb.2016.05.006] [PMID]

22. Garg AX, Adhikari NK, McDonald H, Rosas-Arellano MP, Devereaux PJ, Beyene J, et al. Effects of computerized clinical decision support systems on practitioner performance and patient outcomes: A systematic review. JAMA. 2005; 293(10):1223-38. [DOI:10.1001/jama.293.10.1223] [PMID]

23. Langness S, Ward E, Halbach J, Lizardo R, Davenport K, Bickler $\mathrm{S}$, et al. Plasma D-dimer safely reduces unnecessary $\mathrm{CT}$ scans obtained in the evaluation of pediatric head trauma. Journal of Pediatric Surgery. 2018; 53(4):752-7. [PMID]

24. Papa L, Mittal M, Ramirez J, Silvestri S, Giordano P, Braga C, et al. Neuronal biomarker ubiquitin C-terminal hydrolase detects traumatic intracranial lesions on computed tomography in children and youth with mild traumatic brain injury. Journal of Neurotrauma. 2017; 34(13):2132-40. [DOI:10.1089/neu.2016.4806] [PMID] [PMCID]

25. Manzano S, Holzinger IB, Kellenberger CJ, Lacroix L, Klima Lange $D$, Hersberger $M$, et al. Diagnostic performance of $\mathrm{S} 100 \mathrm{~B}$ protein serum measurement in detecting intracranial injury in children with mild head trauma. Emergency Medicine Journal. 2016; 33(1):42-6. [DOI:10.1136/ emermed-2014-204513] [PMID]

26. Glushakova OY, Glushakov AV, Mannix R, Miller ER, Valadka AB, Hayes RL. The use of blood-based biomarkers to improve the design of clinical trials of traumatic brain injury. In: Skolnick B, Alves WM, editors. Handbook of Neuroemergency Clinical Trials. Cambridge, Massachusetts: Academic Press; 2018. [DOI:10.1016/B978-0-12-804064-5.00008-4]

27. Bouvier $D$, Fournier $M$, Dauphin JB, Amat $F$, Ughetto $S$, Labbé $A$, et al. Serum S100B determination in the management of pediatric mild traumatic brain injury. Clinical Chemistry. 2012; 58(7):1116-22. [DOI:10.1373/ clinchem.2011.180828] [PMID]

28. Papa L, Ramia MM, Kelly JM, Burks SS, Pawlowicz A, Berger RP. Systematic review of clinical research on biomarkers for pediatric traumatic brain injury. Journal of Neurotrauma. 2013; 30(5):324-38. [DOI:10.1089/neu.2012.2545] [PMID] 
This Page Intentionally Left Blank 